\title{
Kelly McKennon
}

The multiplication functions of Kučera

Czechoslovak Mathematical Journal, Vol. 29 (1979), No. 2, 169-177

Persistent URL: http://dml.cz/dmlcz/101594

\section{Terms of use:}

(C) Institute of Mathematics AS CR, 1979

Institute of Mathematics of the Czech Academy of Sciences provides access to digitized documents strictly for personal use. Each copy of any part of this document must contain these Terms of use.

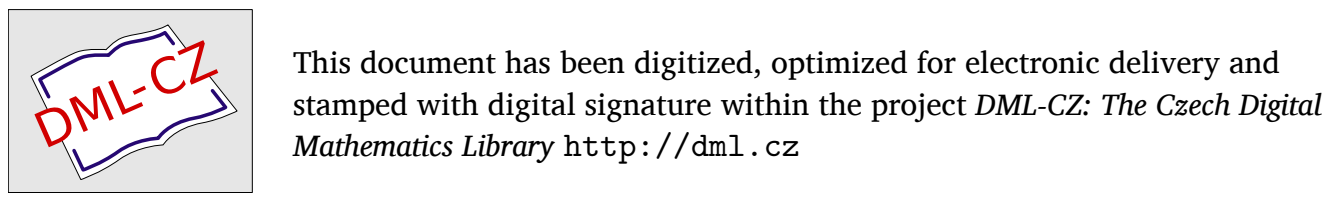




\title{
CZECHOSLOVAK MATHEMATICAL JOURNAL \\ Mathematical Institute of Czechoslovak Academy of Sciences \\ V. 29 (104), PRAHA 25.6.1979, No 1
}

\section{THE MULTIPLICATION FUNCTIONS OF KUCERA}

\author{
Kelly McKennon, Pullman \\ (Received February 25, 1972 in revised form) Way 3,1979
}

In [3] KUCERA introduced some function spaces, the multiplication functions of which have proved useful in proving the continuity of multiplication temperate distributions with slowly increasing functions (see [5] and [6]). It is the purpose of this paper to study these Kucera multiplication functions more closely. We shall prosecute this purpose by first studying multiplication operators on Sobolev Spaces and then we shall characterize the Kucera multiplication functions in terms of the Sobolev operators.

Terminology in this paper will be as in [8].

We shall distinguish from one-another the terms multiplier, multiplication distribution, and multiplication function. Let $A$ and $B$ be translation-invariant Hilbert spaces of locally integrable functions on $R^{n}$. Suppose further that the space $\subseteq$ of rapidly decreasing functions is a dense subspace in each of $A$ and $B$ and that, in each case, its usual topology is finer than the relativized. Let

$$
\mathfrak{B}(A, B)=\{T \mid A \rightarrow B: T \text { is a bounded linear operator }\} .
$$

For each $x \in R^{n}$, let $\tau_{x}$ be the operator of translation by $x$ :

$$
\left[\tau_{x}(f)\right](y)=f(x+y) \text { for all } y \in R^{n} .
$$

Let the family of multipliers be

$$
\mathfrak{m}(A, B)=\left\{T \in \mathfrak{B}(A, B): T \circ \tau_{x}=\tau_{x} \circ T \text { for all } x \in R^{n}\right\} .
$$

Let the family of multiplication distributions be

$$
\mathfrak{M D}(A, B)=\left\{F \in \mathfrak{S}^{\prime}: \text { for some } T_{F} \in \mathfrak{B}(A, B) \text { and all } f, g \in \mathfrak{S},(5) \text { holds }\right\}
$$

where

$$
F(f g)=\int_{R^{n}} T_{F}(f)(x) g(x) \mathrm{d} x
$$


Let the family of multiplication functions be

(6) $\mathfrak{M} \mathfrak{F}(A, B)=\left\{f \mid R^{n} \rightarrow C\right.$ : the map on $A$ sending $h$ to $f h$ is in $\left.\mathfrak{B}(A, B)\right\}$;

thus, to each $f \in \mathfrak{M} \mathfrak{F}(A, B)$, there exists some $T_{f} \in \mathfrak{B}(A, B)$ such that

$$
f h=T_{f}(h) \text { for all } h \in A .
$$

Henceforth $p$ and $q$ will be fixed, non-negative integers. Let $\mathfrak{M}(p)$ be the set of all multi-indices $\alpha$ such that $|\alpha| \leqq p$. The Sobolev Space $W^{p}$ is the linear space

$$
\left\{f \in L_{2}\left(R^{n}\right): D^{\alpha} f \in L_{2}\left(R^{n}\right) \text { for all } \alpha \in \mathfrak{M}(p)\right\}
$$

and

$$
\|f\|_{p}=\left(\sum_{\alpha \in \mathfrak{M}(p)} \int_{R^{n}}\left|D^{\alpha} f(x)\right|^{2} \mathrm{~d} x\right)^{1 / 2}
$$

defines a norm on $W^{p}$ rendering it a Hilbert space; note that $W^{0}$ is just $L_{2}\left(R^{n}\right)$ and \|\|$_{0}$ its usual norm. The space $\hat{W}^{p}$ is the set

$$
\left\{f \in L_{2}\left(R^{n}\right):\|f\|_{p}<\infty\right\}
$$

where

$$
\|f\|_{p}=\left(\int_{R^{n}}\left(1+|x|^{2}\right)^{p}|f(x)|^{2} \mathrm{~d} x\right)^{1 / 2}
$$

for all $f \in L_{2}\left(R^{n}\right)$. As is well-known, $\subseteq$ is a dense subspace of both $W^{p}$ and $\hat{W}^{p}$ and the relativized topology in each case is coarser that the usual topology on $\subseteq$. This of course means that $C_{0}^{\infty}$ is dense in $W^{p}$ and $\hat{W}^{p}$ as well. It is also well-known that the Fourier transform is a topological isomorphism of $W^{p}$ onto $\hat{W}^{p}$ (see [2], Chapter II).

Because of the frequency of its appearance in the sequel, we define the function $\varrho \mid R^{n} \rightarrow R:$

$$
\varrho(x)=\left(1+|x|^{2}\right)^{1 / 2} \text { for all } x \in R^{n} .
$$

It is easy to see that, for all $y, z \in R^{n}$,

$$
1+|z+y|^{2} \leqq\left(1+|z|^{2}\right)(1+|y|)^{2} .
$$

If we let

$$
\sigma(x)=1+|x| \text { for all } x \in R^{n}
$$

then (13) implies

$$
\varrho(z-y) \leqq \varrho(z) \cdot \sigma(y) \text { for all } y, z \in R^{n} .
$$


From (15), we obtain

$$
\varrho(z) \leqq \varrho(z-y) . \sigma(y) \text { for all } y, z \in R^{n} .
$$

The space $\mathrm{m}\left(W^{p}, W^{q}\right)$ was studied in [7]. We shall presently investigate $\mathrm{m}\left(\hat{W}^{p}, \hat{W}^{q}\right)$.

Theorem 1. For each $F \in \mathfrak{M D}\left(W^{p}, W^{q}\right)$, there exists just one operator $C_{F} \in$ $\in \mathrm{m}\left(\hat{W}^{p}, \hat{W}^{q}\right)$ such that

$$
C_{F}(f)=\hat{F} * f \text { for all } f \in S .
$$

Furthermore, the map $C \mid \mathfrak{M D}\left(W^{p}, W^{q}\right) \rightarrow \mathfrak{m}\left(\hat{W}^{p}, \hat{W}^{q}\right)$ is a surjective isomorphism.

Proof. Let $F$ be in $\mathfrak{M D}\left(W^{p}, W^{q}\right)$. Define $T \mid \hat{W}^{p} \rightarrow \hat{W}^{q}$ by letting

$$
T(\hat{f})=T_{F}(f)^{\wedge} \text { for all } f \in W^{p} .
$$

Let $y$ be in $R^{n}$ and define $h_{y} \mid R^{n} \rightarrow C$ by

$$
h_{y}(x)=e^{-i\langle y, x\rangle} \text { for all } x \in R^{n} .
$$

From (5) it is evident that

$$
T_{F}\left(h_{y}, f\right)=h_{y} \cdot T_{F}(f) \text { for all } f \in \mathcal{S} .
$$

Taking the Fourier transform of the above, we obtain

$$
\begin{aligned}
& \tau_{y} \circ T(\hat{f})=\tau_{y} \circ T_{F}(f)^{\wedge}=\left(h_{y} \cdot T_{F}(f)\right)^{\wedge}= \\
& =\left(T_{F}\left(h_{y} \cdot f\right)\right)^{\wedge}=T\left(\left(h_{y} \cdot f\right)^{\wedge}\right)=T \circ \tau_{y}(\hat{f})
\end{aligned}
$$

for all $f \in \mathfrak{S}$. Since $T$ is in $\mathfrak{B}\left(\hat{W}^{p}, \hat{W}^{q}\right)$ and $\mathfrak{S}=\{\hat{f}: f \in \mathfrak{S}\}$ is dense in both $W^{p}$ and $\hat{W}^{p}$, the above shows that $T$ is in $\mathrm{m}\left(\hat{W}^{p}, \hat{W}^{q}\right)$.

Now let $T$ be an arbitrary element of $\mathrm{m}\left(\hat{W}^{p}, \hat{W}^{q}\right)$. A standard argument (see, for example, [8] VI. 3, Theorem 2) shows that

$$
H(f)=[T(f)](0) \text { for all } f \in \mathfrak{S}
$$

defines a temperate distribution $H$ such that

$$
H * f=T(f) \text { for all } f \in \subseteq \text {. }
$$

Let $F$ be the inverse Fourier transform of $H$. By [8] VI. 3, Theorem 6, we have

$$
H * \hat{f}=T_{F}(f)^{\wedge} \text { for all } f \in \mathcal{S} .
$$

That the map $C$ is well-defined follows from the first paragraph of this proof, (17), (18), and (19). That $C$ is surjective follows from (18) and (19). The other assertions of Theorem 1 are trivial. Q.E.D. 
Just what distributions are in $\mathfrak{M D}\left(W^{p}, W^{q}\right)$ seems to be a difficult question. The complete answer for $p=q=0$ is well-known:

$$
\begin{gathered}
L_{\infty}\left(R^{n}\right)=\mathfrak{M} \mathfrak{F}\left(W^{0}, W^{0}\right) \text { and the distributions in } \mathfrak{M D}\left(W^{0}, W^{0}\right) \\
\text { are precisely those associated with the functions in } L_{\infty}\left(R^{n}\right) .
\end{gathered}
$$

We do have some additional information, however, in Theorems 2 and 3 below.

Theorem 2. Let $f \in L_{\infty}\left(R^{n}\right)$ be such that $D^{\alpha} f$ is in $L_{\infty}\left(R^{n}\right)$ for $\alpha \in \mathfrak{M}(q)$. Then $f$ is in $\mathfrak{M} \mathfrak{F}\left(W^{p}, W^{q}\right)$ when $p \geqq q$.

Proof. That $f$ is in $\mathfrak{M} \mathfrak{F}\left(W^{p}, W^{q}\right)$ is obvious upon applying Leibnitz' formula to $f g$ for $g \in W^{p}$.

Theorem 3. If $p<q$, then $\mathfrak{M D}\left(W^{p}, W^{q}\right)=\{0\}$.

Proof. In view of Theorem 1 , it will suffice to show that $m\left(\hat{W}^{p}, \hat{W}^{q}\right)=\{0\}$. Assume that there exists a non-trivial $T \in \mathfrak{m}\left(\hat{W}^{p}, \hat{W}^{q}\right)$ and choose $f \in C_{0}^{\infty}$ such that $T(f) \neq 0$. Let

$$
r=\sup \{|x|: f(x) \neq 0\}
$$

and write $N$ for the set of positive integers. For each $n \in N$, let $x(n)$ be the element of $R^{n}$ of which each coordinate quals $n$. For each $n \in N$, let

$$
f_{n}=\left(\frac{1}{\varrho(x(n))}\right)^{p} \tau_{x(n)} \circ f .
$$

For each $n$ in $N,(11)$ implies

by $(15)$

$$
\begin{gathered}
\left\|f_{n}\right\|_{p}=\left(\frac{1}{\varrho(x(n))}\right)^{p}\left(\int_{R^{n}} \mid f\left(y+\left.x(n)\right|^{2} \cdot \varrho(y)^{2 p} \mathrm{~d} y\right)^{1 / 2}=\right. \\
=\left(\frac{1}{\varrho(x(n))}\right)^{p}\left(\int_{R^{n}}|f(y)|^{2} \cdot \varrho(y-x(n))^{2 n} \mathrm{~d} y\right)^{1 / 2} \leqq
\end{gathered}
$$

by $(21)$

$$
\leqq\left(\frac{1}{\varrho(x(n))}\right)^{p}\left(\int_{R^{n}}|f(y)|^{2} \cdot \sigma(y)^{2 p} \cdot \varrho^{2 p}(x(n)) \mathrm{d} y\right)^{1 / 2} \leqq
$$

$$
\leqq\left(\int_{R^{n}}|f(y)|^{2} \cdot \sigma(y)^{2 p} \mathrm{~d} y\right)^{1 / 2} .
$$

Since $\sigma<1+\varrho$, this implies $\sigma^{2 p} \leqq(2 p+1) \varrho^{2 p}$ so that

$$
\sup _{n \in N}\left\|f_{n}\right\|_{p} \leqq(2 p+1)^{1 / 2}\|f\|_{p}<\infty .
$$


Now choose some $m>0$ such that

$$
M=\int_{|y| \leqq m}|[T(f)](y)|^{2} \mathrm{~d} y>0 .
$$

From (16) and the fact that $\varrho$ is even, we have

$$
\varrho(y-x(n)) \geqq \frac{\varrho(x(n))}{\sigma(y)}
$$

for all $y \in R^{n}$ and $n \in N$. For each $n \in N$, we have

$$
\begin{gathered}
\left\|T\left(f_{n}\right)\right\|_{\hat{q}}=\left\|T\left(\frac{1}{\varrho(r)}\right)^{p} \tau_{x(n)}(f)\right\|_{q}^{\wedge}=\left(\frac{1}{\varrho(x(n))}\right)^{p}\left\|\tau_{x(n)} \circ T(f)\right\|_{\hat{q}}^{\wedge}= \\
=\left(\frac{1}{\varrho(x(n))}\right)^{p}\left(\int_{R^{n}}|[T(f)](y+x(n))|^{2} \cdot \varrho(y)^{2 q} \mathrm{~d} y\right)^{1 / 2} \geqq \\
\geqq\left(\frac{1}{\varrho(x(n))}\right)^{p}\left(\int_{|y| \leqq m}|[T(f)](y)|^{2} \cdot \varrho(y-x(n))^{2 q} \mathrm{~d} y\right)^{1 / 2} \geqq
\end{gathered}
$$

by $(23)$

$$
\geqq\left(\frac{1}{\varrho(x(n))}\right)^{p}\left(\int_{|y| \leqq m}|[T(f)](y)|^{2} \cdot\left(\frac{\varrho\left(x_{n}\right)}{\sigma(y)}\right)^{2 q} \mathrm{~d} y\right)^{1 / 2} \geqq
$$

by $(14)$

$$
\left(\frac{1}{1+m}\right)^{q}\left(\frac{1}{\varrho(x(n))}\right)^{p} M^{1 / 2} \cdot \varrho^{q}\left(x_{n}\right) .
$$

Since $\lim \varrho\left(x_{n}\right)=\infty$, it follows that

$$
\lim _{n}\left\|T\left(f_{n}\right)\right\|_{\hat{q}}^{\wedge}=\infty .
$$

This, with (22), shows that $T \notin \mathfrak{B}\left(\hat{W}^{p}, \hat{W}^{q}\right)$ : a contradiction. Q.E.D.

We now turn to the Kucera spaces, which are linear subspaces of the Sobolev spaces. Let $\mathfrak{P}(p)$ be the set of all pairs $(\alpha, \beta)$ such that $\alpha, \beta, \alpha+\beta \in \mathfrak{M}(p)$. The Kucera space $H^{p}$ is the set

$$
\left\{f \in W^{p}:\left|\|f \mid\|_{p}<\infty\right\}\right.
$$

where

$$
\|f \mid\|_{p}=\left(\sum_{(\alpha, \beta) \in \mathfrak{B}(p)} \int_{R^{n}}\left|x^{\alpha} \cdot D^{\beta} f(x)\right|^{2} \mathrm{~d} x\right)^{1 / 2}
$$

for each $f \in W^{p}$. Under the norm \|\|$\|_{p}, H^{p}$ is a Hilbert space. It is known that the 
Fourier transform is a topological isomorphism of $H^{p}$ onto itself and that $\mathfrak{S}$ is dense in $H^{p}$ (see [3]). The notation $\mathfrak{D}_{p, q}$ is usually used instead of $\mathfrak{M} \mathfrak{F}\left(H^{p}, H^{q}\right)$.

Theorem 4. For each $F \in \mathfrak{M D}\left(H^{p}, H^{q}\right)$, there exists just one operator $C_{F} \in \mathfrak{m}\left(H^{p}, H^{q}\right)$ such that

$$
C_{F}(f)=\hat{F} * f \text { for all } f \in S \text {. }
$$

Furthermore, the map $F \rightarrow C_{F}$ is onto $\mathrm{m}\left(H^{p}, H^{q}\right)$.

Proof. Repeat the proof of Theorem 1 , replacing $W^{p}$ and $\hat{W}^{p}$ by $H^{p}$, and $W^{p}$ and $\hat{W}^{q}$ by $H^{q}$.

Lemma 1. Let $T$ be in $\mathfrak{m}\left(H^{p}, H^{q}\right)$. Then there exists a family $\left\{T_{\alpha, \beta}:(\alpha, \beta) \in \mathfrak{P}(p)\right\}$ such that

$$
\text { for each }(\alpha, \beta) \in \mathfrak{P}(p), \quad T_{\alpha, \beta} \in \mathfrak{m}\left(\hat{W}^{|\alpha|}, H^{q}\right)
$$

$$
T=\sum_{(\alpha, \beta) \in \mathfrak{P}(p)} T_{\alpha, \beta} \circ D^{\beta} .
$$

Proof. For each $(\alpha, \beta) \in \mathfrak{P}(p)$, let $A_{\alpha, \beta}$ be the Hilbert space $\hat{W}^{|\alpha|}$ and let $A$ be the product Hilbert space $\prod_{(\alpha, \beta) \in \mathfrak{F}(p)} A_{\alpha . \beta}$. The norm \|\|$_{A}$ on $A$ is given by

$$
\|f\|_{A}=\left[\sum_{(\alpha, \beta) \in \mathfrak{F}(p)}\left(\left\|f_{(\alpha, \beta)}\right\|_{|\alpha|}\right)^{2}\right]^{1 / 2}
$$

for all $f \in A$. Define the operator $\Psi \mid H^{p} \rightarrow A$ by, for each $f \in H^{p}$, letting

$$
\Psi(f)_{(\alpha, \beta)}=D^{\beta}(f) \text { for all }(\alpha, \beta) \in \mathfrak{P}(p) .
$$

Then $\Psi$ is evidently a topological isomorphism. Since $H^{p}$ is complete, it follows that $\Psi\left(H^{p}\right)$ is a closed subspace of $A$ and so $A$ is exactly the direct sum $\Psi\left(H^{p}\right) \oplus \Psi\left(H^{p}\right)^{\perp}$. Define the operator $V \mid A \rightarrow H^{q}$ by letting

$$
V(f+g)=T_{\circ} \Psi^{-1}(f)
$$

for all $f \in \Psi\left(H^{p}\right)$ and $g \in \Psi\left(H^{p}\right)^{\perp}$. Thus, $V$ is in $\mathfrak{B}\left(A, H^{q}\right)$ and

$$
V \circ \Psi=T \text {. }
$$

Since the differential operators $D^{\beta}$ commute with translations, so does $\Psi$. Since $H^{p}$ is translation invariant, so is $\Psi\left(H^{p}\right)$ and so $\Psi\left(H^{p}\right)^{\perp}$ as well. By (27), we have

$$
\begin{aligned}
& V \circ \tau_{x}(f+g)=V\left(\tau_{x}(f)+\tau_{x}(g)\right)=T \circ \Psi^{-1}\left(\tau_{x}(f)\right)= \\
& =T\left(\tau_{x}\left(\Psi^{-1}(f)\right)\right)=\tau_{x} \circ T \circ \Psi^{-1}(f)=\tau_{x} \circ V(f+g)
\end{aligned}
$$


for all $f \in \Psi\left(H^{p}\right), g \in \Psi\left(H^{p}\right)^{\perp}$, and $x \in R^{n}$. Thus

$$
V \in \mathfrak{m}\left(A, H^{q}\right) .
$$

For each $(\alpha, \beta) \in \mathfrak{P}(p)$, let $I_{\alpha, \beta}$ be the canonical imbedding of $A_{\alpha, \beta}$ into $A$ and let

$$
T_{\alpha, \beta}=V_{\circ} I_{\alpha, \beta}
$$

for each $(\alpha, \beta) \in \mathfrak{P}(p)$. Since $I_{\alpha, \beta}$ commutes with translations, it follows that (i) holds. For each $f \in H^{p}$, we have from (28), (26), and (30),

$$
\begin{aligned}
& T(f)=V \circ \Psi(f)=V\left(\sum_{(\alpha, \beta) \in \mathfrak{P}(p)} I_{\alpha, \beta}\left(\Psi(f)_{\alpha, \beta}\right)=\right. \\
& =\sum_{(\alpha, \beta) \in \mathfrak{P}(p)} V \circ I_{\alpha, \beta} \circ D^{\beta}(f)=\sum_{(\alpha, \beta) \in \mathfrak{P}(p)} T_{\alpha, \beta} \circ D^{\beta}(f)
\end{aligned}
$$

which proves (ii). Q.E.D.

Lemma 2. A linear operator $T$ from $\hat{W}^{q}$ into $\hat{W}^{p}$ is in $\mathrm{m}\left(\hat{W}^{q}, H^{p}\right)$ if and only if, for all $(\alpha, \beta) \in \mathfrak{P}(p)$,

$$
D^{\alpha} \circ T \in \mathfrak{m}\left(\hat{W}^{q}, \hat{W}^{|\beta|}\right) .
$$

Proof. Suppose first that $T \in \mathfrak{m}\left(W^{q}, H^{p}\right)$ and let $(\alpha, \beta)$ be in $\mathfrak{P}(p)$. It is evident from $(22)$ that $D^{\alpha}$ is in $B\left(H^{q}, W^{|\beta|}\right)$. Since $D^{\alpha}$ commutes with translations, it is in $\mathrm{m}\left(H^{q}, W^{|\beta|}\right)$ as well. It follows that (i) holds.

Now suppose that (i) holds for all $(\alpha, \beta) \in \mathfrak{P}(p)$. Let the spaces $A_{\alpha, \beta}$ and $A$ be as in the proof of Lemma 1. Consider the topological isomorphism $\Psi$ from $H^{p}$ onto $A$ which was constructed the proof of Lemma 1 . Note that, for each $(\alpha, \beta) \in \mathfrak{P}(p)$, the projection $\pi_{\alpha, \beta}$ of $A$ onto $A_{\alpha, \beta}$ satisfies

$$
\pi_{\alpha, \beta} \circ \Psi=D^{\alpha} .
$$

It is a consequence of (i) that $T\left(\hat{W}^{q}\right) \subset H^{p}$. This, implies

$$
\pi_{\alpha, \beta} \circ \Psi \circ T=D^{\alpha} \circ T \text { for all }(\alpha, \beta) \in \mathfrak{P}(p) .
$$

Since a map into a prcduct space is continuous if its composition with each canonical projection is continuous, (i) and (32) imply that $\Psi$ 。T is continuous. If we define $\Psi^{-1}$ on $\Psi\left(H^{p}\right)$, we have $T=\Psi^{-1} \circ \Psi \circ T$ and so $T$ is in $\mathfrak{B}\left(\hat{W}^{q}, H^{p}\right)$. Recalling that $\Psi$, and thus $\Psi^{-1}$, commute with translations, we see that, for each $x \in R^{n}$ and $f \in \hat{W}^{q}$,

$$
T \circ \tau_{x}(f)=\Psi^{-1} \circ \Psi\left(T \circ \tau_{x}(f)\right)=
$$

by (32)

$$
=\Psi^{-1}\left(\sum_{(\alpha, \beta) \in \mathfrak{R}(p)} \pi_{\alpha, \beta} \circ \Psi \circ T \circ \tau_{x}(f)\right)=\Psi^{-1}\left(\sum_{(\alpha, \beta) \in \mathfrak{P}(p)} D^{\alpha} \circ T\left(\tau_{x}(f)\right)\right)=
$$


by (i) and (32)

$$
\begin{gathered}
=\Psi^{-1}\left(\sum_{(\alpha, \beta) \in \mathfrak{P}(p)} \tau_{x} \circ D^{\alpha} \circ T(f)\right)=\Psi^{-1} \circ \tau_{x}\left(\sum_{(\alpha, \beta) \in \mathfrak{P}(p)} \pi_{\alpha, \beta} \circ \Psi \circ T(f)\right)= \\
=\tau_{x} \circ \Psi \circ T(f)=\tau_{x} \circ T(f) .
\end{gathered}
$$

Thus $T$ is in $m\left(\hat{W}^{q}, H^{p}\right)$. Q.E.D.

Theorem 5. Let $T$ be an arbitrary multiplier in $\mathrm{m}\left(H^{p}, H^{q}\right)$. Then there exists a family $\left\{T_{\alpha, \beta}:(\alpha, \beta) \in \mathfrak{P}(p)\right\}$ such that

$$
\begin{gathered}
T_{\alpha, \beta} \in \mathrm{m}\left(\hat{W}^{|\beta|}, \hat{W}^{q}\right) \quad \text { for all }(\alpha, \beta) \in \mathfrak{P}(p) ; \\
D^{\gamma} \circ T_{\alpha, \beta} \in \mathrm{m}\left(\hat{W}^{|\beta|}, \hat{W}^{|\delta|}\right) \text { for all }(\gamma, \delta) \in \mathfrak{P}(q) ; \\
T=\sum_{(\alpha, \beta) \in \mathfrak{P}(p)} T_{\alpha, \beta} \circ D^{\alpha} .
\end{gathered}
$$

Furthermore, if (i), (ii) and (iii) hold, then $T$ is in $\mathrm{m}\left(H^{p}, H^{q}\right)$.

Pro of. Suppose first that $T$ is in $\mathfrak{m}\left(H^{p}, H^{q}\right)$. Choose the family $\left\{T_{\alpha, \beta}:(\alpha, \beta) \in \mathfrak{P}(p)\right\}$ as in Lemma 1. Then (i) holds, because $H^{q}$ is a subset of $\hat{W}^{q}$ and the topology on $H^{q}$ is finer than that relativized from $\hat{W}^{q}$. Since (iii) holds from Lemma 1 , we need only show (ii). Fix $(\alpha, \beta)$ in $\mathfrak{P}(p)$. Then $T_{\alpha, \beta}$ is in $\mathfrak{m}\left(\hat{W}^{|\beta|}, H^{q}\right)$ and, if we apply Lemma 2 , we obtain (ii).

Now suppose that (i), (ii), and (iii) hold where $T$ has $H^{p}$ as its domain. Fix $(\alpha, \beta)$ in $\mathfrak{P}(p)$. Lemma 2 and (ii) imply that $T_{\alpha, \beta}$ is in $\mathrm{m}\left(\hat{W}^{|\beta|}, H^{q}\right)$. Note that $D^{\alpha}$ is in $\mathfrak{m}\left(H^{p}, \hat{W}^{|\beta|}\right)$. It follows that $T_{\alpha, \beta} \circ D^{\alpha}$ is in $\mathfrak{m}\left(H^{p}, H^{q}\right)$. Hence, from (iii), $T$ is in $\mathrm{m}\left(H^{p}, H^{q}\right)$. Q.E.D.

Corollary 1. Let $T$ and $\left\{T_{\alpha, \beta}:(\alpha, \beta) \in \mathfrak{P}(p)\right\}$ be as in Theorem 5. Then $T_{\alpha, \beta}=0$ for all $\beta \in \mathfrak{M}(q-1)$.

Proof. This corollary follows directly from Theorem 3 and Theorem 5. Q.E.D. Putting together Theorem 4, Theorem 5, and the corollary to Theorem 5, we obtain

Theorem 6. Let $F$ be in $\mathfrak{M D}\left(H^{p}, H^{q}\right)$. Then there exists a family $\left\{F_{\alpha, \beta}:(\alpha, \beta) \in\right.$ $\in \mathfrak{P}(p),|\beta| \geqq q\}$ such that

$$
\begin{gathered}
F_{\alpha, \beta} \in \mathfrak{M D}\left(H^{|\beta|}, H^{q}\right) \quad \text { for all }(\alpha, \beta) \in \mathfrak{P}(p) ; \\
x^{\gamma} \cdot F_{\alpha, \beta} \in \mathfrak{M D}\left(H^{|\beta|}, H^{|\delta|}\right) \text { for all }(\gamma, \delta) \in \mathfrak{P}(q) ; \\
F=\sum_{(\alpha, \beta)} F_{\alpha, \beta} \cdot x^{\alpha} .
\end{gathered}
$$


Conversely, if $F$ is defined by (iii), then $F \in \mathfrak{M D}\left(H^{p}, H^{q}\right)$.

Corollary 2. If $p<q$, then $\mathfrak{M D}\left(H^{p}, H^{q}\right)=\{0\}$.

Proof. In view of Theorem 3, this corollary is trivial.

Corollary 3. Let $\alpha$ be in $\mathfrak{M}(p-q)$ and let $f$ be any function in $L_{\infty}\left(R^{n}\right)$ such that (i)

$$
x^{\gamma} D^{\delta} f \in L_{\infty}\left(R^{n}\right) \text { for all }(\gamma, \delta) \in \mathfrak{P}(q) \text {. }
$$

Then, if

$$
h(x)=x^{\alpha} f(x) \text { for all } x \in R^{n},
$$

$h$ is in $\mathfrak{M} \mathfrak{F}\left(H^{p}, H^{q}\right)=\mathfrak{D}_{p, q}$.

Proof. Let $\beta \in \mathfrak{M}(p)$ be such that $(\alpha, \beta) \in \mathfrak{P}(p)$. That $x^{\gamma} f$ is in $\mathfrak{M F}\left(H^{|\beta|}, H^{|\delta|}\right)$ for all $(\gamma, \delta) \in \mathfrak{P}(q)$ follows from Theorem 2 and an application of Leibnitz' formula to (i). That Corollary 3 holds, now follows upon viewing $\mathfrak{M} \mathfrak{F}\left(H^{p}, H^{q}\right)$ as a subspace of $\mathfrak{M P}\left(H^{p}, H^{q}\right)$ and applying Theorem 6 to $T_{\alpha, \beta}=f$. Q.E.D.

Improvement of Corollary 3 seems to be dependebt on improvement of Theorem 2. Corollary 3 is equivalent to Proposition 8 in [4], which may be proved by direct application of Leibnitz' formula. We have included our proof to suggest an avenue to extending Corollary 3.

\section{Bibliography}

[1] Hewitt, E. and Ross, K.: Abstract Harmonic Analysis, Vol. 1. Berlin: Springer Verlag. 1963.

[2] Hörmander, L.: Linear Partial Differential Operators. Berlin: Springer Verlag. 1969.

[3] Kucera, J.: Fourier $\mathrm{L}_{2}$-transform of distributions. Czech. Math. J. 19, 143-153 (1969).

[4] Kucera, J.: On multipliers of temperate distributions. Czech. Math. J. 21, 610-618 (1971).

[5] Kucera, $J$. and McKennon, K.: Certain topologies on the space of temperate distributions and its multipliers. Indiana Math. J. 24, 773- 775 (1975).

[6] Kucera, J. and McKennon, K.: The topology on certain spaces of multipliers. Rocky Mountain J. of Math. 7, 377-383 (1977).

[7] McKennon, K.: Sobolev Multipliers. To appear in Czech. Math. J.

[8] Yosida, K.: Functional Analysis. Berlin: Springer Verlag. 1968.

Author's address: Department of Mathematics, Washington State University, Pullman, Washington 99163, U.S.A. 